\title{
Conocimiento didáctico del contenido en química orgánica: Estudio de caso de un profesor universitario
}

\author{
Pedagogical Content Knowledge in Organic Chemistry: A Case Study of the University Teacher
Conhecimento didático do conteúdo em química orgânica: estudo de caso de um professor universitário

\author{
Diana Lineth Parga-Lozano ${ }^{7}$ \\ Universidad Pedagógica Nacional \\ Bogotá, Colombia \\ dparga@pedagogica.edu.co \\ (iD) http://orcid.org/0000-0002-7899-0767 \\ William Fernando Moreno-Torres ${ }^{2}$ \\ Secretaría de Educación de Cundinamarca \\ Bogotá, Colombia \\ elpostigio435@gmail.com \\ iD http://orcid.org/0000-0003-3434-754X
}

Recibido 15 de abril de 2016 • Corregido 29 de julio de 2017 • Aceptado 25 de agosto de 2017

Received 15 de abril de 2016 • Revised 29 julio 2017 • Accepted 25 agosto 2017

Recebido 15 de abril de 2016 • Revisado 29 de julio 2017 • Aprovado 25 de agosto de 2017

\begin{abstract}
Resumen: A partir de la didáctica de las ciencias, la línea sobre el conocimiento didáctico del contenido o CDC hace aportes importantes para analizar la formación del profesorado y la mejora profesional. En este sentido, se realizó una investigación para caracterizar el CDC de un profesor, licenciado en química, que enseña conceptos de química orgánica en una institución de educación superior. El estudio fue de naturaleza cualitativa, con diseño de estudio de caso; como técnica de análisis, se usó el análisis documental; para recolectar la información, se usaron instrumentos como representaciones de contenido, repertorios profesionales de experiencia didáctica, análisis documental de las producciones del profesor participante, observaciones de clase y entrevistas. Lo encontrado para este
\end{abstract}

\footnotetext{
1 Es licenciada en biología y química, magíster en docencia de la química; doctoranda del programa de posgraduación de Educación en ciencias en la UNESP, Bauru, SP, Brasil. Es profesora del Departamento de Química en la Universidad Pedagógica Nacional (UPN) en Bogotá, en los programas de Licenciatura en Química y Maestría en Docencia de la Química. Es investigadora asociada, en didáctica de la química en el grupo Alternaciencias: Alternativas para la enseñanza de las ciencias. Ha publicado libros de texto para la enseñanza de las ciencias naturales (biología y química) y artículos en líneas relacionadas con el conocimiento didáctico del contenido, CTSA y ambientalización curricular.

${ }^{2}$ Es licenciado en química, magíster en docencia de la química. Es profesor de química en la Secretaría de Educación de Cundinamarca, Colombia; cuenta con experiencia laboral en el sector educativo privado y público.
} 
caso muestra que el profesor se ve como un químico y no como un profesor de química, no tiene identidad profesional, tal vez por ello predomina en él su conocimiento disciplinar y desconoce lo relacionado con los conocimientos del contexto escolar, histórico-epistemológico y psicopedagógico. Lo anterior evidencia, como característica del CDC del participante, que este es incipiente, porque sus componentes están desarticulados; lo que determina debilidades al enseñar conceptos de química orgánica. Estos aspectos hacen pensar que es necesario fortalecer en la formación inicial del profesorado su rol como profesional de la docencia y la forma de integrar los componentes del CDC.

Palabras claves: Conocimiento del contexto escolar; conocimiento didáctico del contenido; conocimiento disciplinar; conocimiento histórico-epistemológico; conocimiento psicopedagógico.

\begin{abstract}
From Science Education, the Pedagogical Content Knowledge (PCK) is making an important contribution to the analysis of teacher training and the improvement of professionals. As such, a study was conducted to characterise the PCK of a teacher holding a degree in chemistry teaching; he teaches organic chemistry concepts at a higher education institution. The research involved a case study and was qualitative in nature; document analysis was also used as an analytical technique; besides, information was collected by use of Representations of Content (ReCo), Pedagogical and Professional Experience Repertoires (PaP-eR), document analysis of the participating teacher's work, classroom observation, and interviews. With these tools, we were able to determine the strengths and weaknesses of the participating teacher, and from there, to infer aspects that needed improving in training undergraduates in chemistry. I found that, in this case, the teacher sees himself as chemist and not as chemistry teacher (i.e. there is a lack of professional identity); perhaps, because it is the dominant factor in his disciplinary knowledge at the expense of topics related to the knowledge of school context, as well as historicalepistemological and psycho-pedagogical knowledge. The latter indicates, as a characteristic of the participant's PCK, that this PCK is incipient due to the disarticulation of its components, which weakens the teaching of organic chemistry concepts. This suggests the need to strengthen the initial training of teachers in their role as teaching professionals, and to integrate components of the PCK.
\end{abstract}

Keywords: Contextual school knowledge; pedagogical content knowledge; disciplinary knowledge; historical-epistemological knowledge; psycho-pedagogical knowledge.

Resumo: A partir da educação didática das ciências, a linha sobre o conhecimento didático do conteúdo ou CDC faz importantes contribuições para analisar a formação dos professores e as melhoras como profissional. Neste sentido, foi realizada uma investigação para caracterizar o CDC de um professor, graduado em química, responsável do ensino de conceitos de química orgânica em uma instituição de educação superior. $O$ estudo foi qualitativo, com formato de estudo caso. Como técnica se utilizou a análise documental; os instrumentos usados para buscar informações foram: representações de conteúdo, repertórios profissionais de experiência didáticas, análise documentais das produções do professor participante como também observações em sala de aula e entrevistas. $O$ que foi encontrado para este caso demonstra que o professor é visto como um químico e não como um professor de química, não possui identidade profissional, predominando o seu conhecimento disciplinar sobre os conhecimentos relacionados com o contexto escolar, histórico-epistemológico e pedagógico. Isto tudo evidência, de acordo com a característica do CDC desse professor, que sua prática é deficiente, porque seus componentes educativos estão desarticulados e são determinantes 


\begin{abstract}
para mostrar as fraquezas no ensino dos conceitos de química orgânica. Esses aspectos sugerem a necessidade de reforçar a formação inicial dos professores em sua função como profissional docente e também de que forma integrar os componentes do CDC.
\end{abstract}

Palavras-chave: Conhecimento do contexto escolar; Conhecimento Pedagógico do Conteúdo; conhecimento disciplinar; histórico e conhecimento epistemológico; conhecimento psicopedagógico.

\title{
Introducción
}

La línea de investigación sobre el conocimiento didáctico del contenido (CDC en adelante) se ha considerado fructífera y potente al momento de establecer programas de formación inicial y permanente del profesorado de ciencias, lo cual la ha puesto como referente a ser considerada al evaluar el desempeño profesional del profesorado y el diseño curricular (Mora y Parga, 2014). Este hecho, dicen los autores, ha permitido entender la didáctica de las disciplinas como un campo dedicado a la enseñanza de los contenidos que garantiza su aprendizaje en el estudiantado. Por lo tanto, se han creado procedimientos de formación inicial y permanente del profesorado, que pueden ser interpretados desde visiones como las de la transformación, transposición-destransposición e integración didáctica.

Así, es importante hacer estudios de diagnóstico, reflexión y triangulación de los conocimientos-creencias que refleja el profesorado de química, particularmente en cuanto a sus intencionalidades curriculares para aportar elementos críticos que muestren que la formación inicial y continua del profesorado está generando profesionales idóneos o no, para la profesión docente y que desde los planteamientos del CDC se pueden identificar las capacidadescompetencias profesionales para asumir el reto social de la docencia. En este sentido, se ha hecho el presente artículo que muestra los resultados de un estudio de caso para caracterizar el CDC de un profesor, licenciado en química, que enseña química orgánica en una institución de educación superior, con el propósito de identificar la relación entre los componentes del CDC y su integración o no.

Según lo anterior, es importante precisar que los primeros planteamientos sobre la investigación de los conocimientos que tiene el profesorado para enseñar, fue hecha por Shulman (1986), aunque hoy existen varios planteamientos paralelos; así, por ejemplo, la transposición didáctica de Chevallard (1991), planteada para la didáctica de las matemáticas, se extendió a otras áreas disciplinares; además, definió la didáctica de las matemáticas como una disciplina independiente, tal como sucedió con la didáctica de las ciencias (Garritz, Daza-Rosales y Lorenzo, 2014). La destransposición didáctica de Antibi y Brousseau (2000) es una forma alternativa de transposición interesada en modificar concepciones enseñadas previamente, que pueden ser obstáculo de aprendizajes futuros.

Respecto a la transposición didáctica, Chevallard (1991) explica que se da porque el conocimiento didáctico y el académico, estando relacionados no son superponibles, y que 
doi: http://dx.doi.org/10.15359/ree.21-3.3

URL: http://www.una.ac.cr/educare

CORREO: educare@una.cr

necesariamente el personal didacta debe cuestionar ideas, interrogar sobre las pruebas, tomar distancia sobre el objeto de estudio, en lo que él habla de la "vigilancia epistemológica"; lo que lleva precisamente a que considere los procesos y acciones que permiten elegir los tópicos del conocimiento científico; no es solamente lo que los sujetos expertos condicionan sobre el conocimiento disciplinar sino también los autores y autoras de libro de textos, didactas de la disciplina, los contextos sociales, políticos y culturales. Esta transposición vincula una serie de conocimientos que permiten que el docente escoja, planifique los contenidos y las formas de enseñarlos (Garritz et al., 2014). Respecto a la transposición y la transformación didáctica, Mora y Parga (2014) afirman:

Para muchos la transformación didáctica de Shulman y la transposición didáctica de Chevallard son lo mismo, debido a que estaban planteando metas comunes en el sentido de mejorar la enseñanza, los procesos epistemológicos asociados, la manera de concebirlo y el papel del profesor en la escuela, obedecían a corrientes distintas que generaban resultados con variaciones significativas. (p.107)

Para Mora y Parga (2014), el CDC es más que sumar o mezclar sus componentes; lo definen como producto de una hibridación sistémica y compleja que implica una integración didáctica -y no una transposición o una transformación-, en donde se complejizan los cocimientoscreencias del profesorado y del estudiantado.

Lo que el profesorado piensa y enseña, ayuda a identificar sus creencias y concepciones sobre la filosofía de la ciencia, la enseñanza y el aprendizaje; así el CDC va más allá del conocimiento disciplinar; y permite establecer la diferencia entre la labor docente y la enseñanza que realizan otros grupos profesionales que, sin serlo, se dedican a enseñar, ya que el CDC contiene un saber epistemológico, propio, idiosincrático, diverso, complejo y construido históricamente por cada docente.

Gess-Newsome (1999, citado por Garritz et al., 2014) afirma que el CDC, con sus orientaciones y transformaciones, parece reconocer dinámicas no solo de las acciones del profesorado, sino en relación con los elementos de su conocimiento profesional.

En los años ochenta, cuando Shulman (1986, citado por Parga, Mora y Martínez, 2007) estudia el PCK (Pedagogical Contend Knowledge), plantea que el conocimiento del profesorado está integrado a los aspectos del conocimiento del contenido temático, conocimiento pedagógico del contenido y el conocimiento curricular; por su parte, Mellado (1994) vincula el estudio de las didácticas generales propiciando el CDC; para el presente estudio se aborda la idea del CDC planteado por Mora y Parga $(2008,2014)$ y Parga y Mora (2014). En este sentido, el profesorado expresa la necesidad de transformar los contenidos desde el punto de vista de la comunidad que produce el conocimiento hacia un punto de vista de quien necesita enseñarlos, así emerge un conocimiento nuevo, que articula el contexto escolar, la cotidianidad y el conocimiento científico y metacientífico con la intencionalidad de ser enseñable, lo cual desemboca en la integración de estos conocimientos científicos realizados por el personal 
docente de acuerdo con su intención y su experiencia (Mora y Parga, 2007, 2008, 2014).

EI CDC de cada docente estaría gobernado por patrones de comportamientos variables, pero perceptibles en un periodo de tiempo; en este sentido, el CDC, al centrarse en la enseñabilidad de los contenidos en situaciones problemáticas complejas, está sujeto a mejoras, pero también a bifurcaciones permanentes, las cuales lo desvían de patrones establecidos por rutinas, por lo tanto, las categorías del CDC están abiertas al diálogo tradicionalmente en conflicto entre las ciencias naturales y sociales, las humanidades y los saberes cotidianos y escolares que conforman el sistema de conocimiento-creencias del profesorado (Mora y Parga, 2014).

Siguiendo las ideas de estos autores, el CDC es un sistema complejo, surge de la interacción de cuatro categorías importantes: los conocimientos-creencias de lo disciplinar (CD), lo psicopedagógico (CP), lo contextual (CC) y lo metadisciplinar (CM). Este último implica lo histórico-epistemológico, social, político, tecnológico, entre otros, de la disciplina; en este artículo

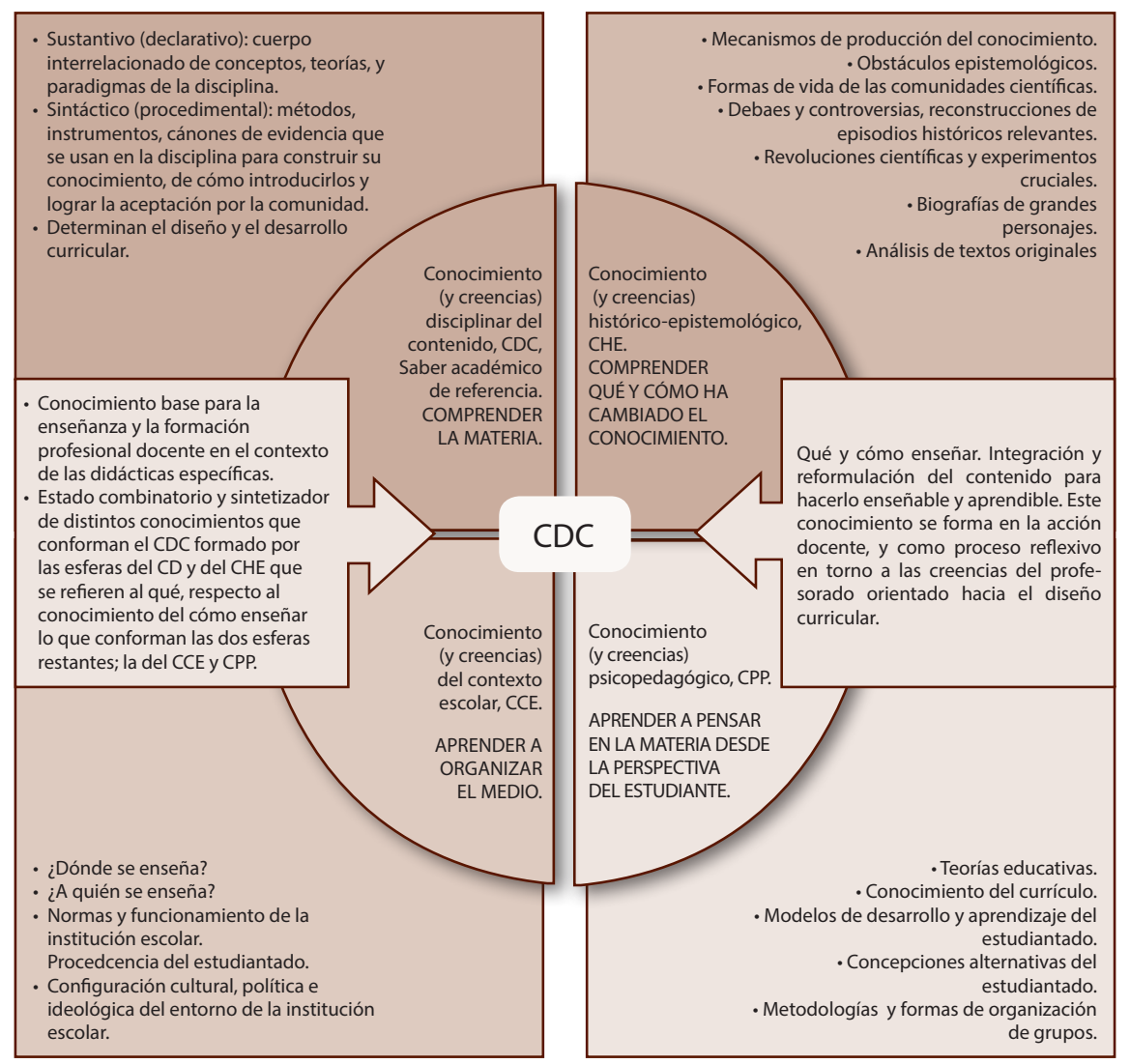

Figura 1: Categorías y componentes del CDC

Nota: Tomado de Mora y Parga (2008, p. 64) 
doi: http://dx.doi.org/10.15359/ree.21-3.3

URL: http://www.una.ac.cr/educare

CORREO: educare@una.cr

lo trataremos como el conocimiento histórico-epistemológico (CHE), por el énfasis dado a estas dos metadisciplinas. La Figura 1 representa los componentes planteados para el CDC.

Para el presente trabajo, se han unificado categorías de conocimientos-creencias que pueden conformar el CDC (Figura1); en concreto el CD, CP, CC y CM: CHE, organizados en diferentes componentes que nacen desde los modelos didácticos de cada docente, sistema que se hibrida según el problema de enseñabilidad de contenidos en el aula, así surge un CDC específico que es compatible con otros docentes cuando hay reflexión en la acción educativa y sobre esta (Mora y Parga, 2014). Estos criterios nos permitieron caracterizar, a través de un estudio de caso con análisis documental, el CDC de un profesor que se ha formado como licenciado en química y que enseña en una institución de educación superior.

\section{Marco teórico}

\section{Reconociendo el CDC del profesorado}

Para comprender el estudio del CDC del profesorado de ciencias de la naturaleza, se han elaborado estrategias para analizar las elaboraciones de las representaciones denominadas representaciones de contenido (ReCo) y los repertorios profesionales de experiencia didáctica (ReP-Ed) planteados inicialmente por Mulhall, Berry y Lourgrhan, (2003), pero con una visión más individual del profesorado; las ReCo y los ReP-Ed se contrastan para ser usados como estrategia para el desarrollo profesional; también se utilizan tópicos relacionados con el conocimiento base y CDC en donde hay: (i) discusión de experiencias de estudiante y de docente, (ii) discusión del conocimiento pedagógico de revistas en torno al concepto, (iii) expresiones de intención de la enseñanza, (iv) enseñanza del tópico en contextos escolares, (v) reflexión entre conocimiento base y CDC (Mora y Parga, 2008). Como lo expresan estos estudios, significa asumir e identificar sus posturas epistemológicas para la enseñanza, hacer sus propios diseños curriculares, hacer investigación pedagógico-didáctica de los procesos de enseñanza aprendizaje en el aula (Mora y Parga, 2005).

\section{El currículo}

Las sugerencias elaboradas por Posner (2004) en su libro Análisis del currículo, fueron un referente para la investigación, dado que es uno de los aspectos en los que se reflejó el CDC del profesorado. Este autor plantea siete conceptos del currículo así: (i) Alcance y secuencia: matriz de objetivos asignado a los grados y agrupados a un tema en común. (ii) Programa de estudios: incluye fundamentos, temas, recursos y evaluación. (iii) Esquema de contenido: lista de tópicos organizados como un esquema. (iv) Estándares: lista de conocimientos y habilidades requeridos al terminar el curso. (v) Libros de texto: materiales educativos usados como guías de enseñanza 
en el salón de clases. (vi) Ruta de estudio: serie de cursos que el estudiantado debe completar. (vii) Experiencias planeadas: experiencias académicas, atléticas, emocionales o sociales y que han sido planeadas en la escuela.

De acuerdo con la intencionalidad del presente trabajo, la mejor representación del modelo curricular dominante está en el trabajo de Tyler (1949, citado por Posner, 2004), quien planteó cuatro preguntas fundamentales: ¿Cuáles propósitos educativos debe tratar de alcanzar la escuela; qué experiencias educativas deben ofrecerse para alcanzar estos propósitos; cómo se organizan estas experiencias de manera eficaz; y cómo se determina si se han alcanzado estos objetivos? Sin embargo, se cuestiona que en esta propuesta los recursos didácticos no son enunciados de forma explícita, lo que es importante en el modelo curricular.

Cuando las decisiones se consideran técnicas, el propósito de la escuela es promover o producir conocimiento, así Posner (2004) propone los marcos conceptuales de la producción técnica orientados de la siguiente forma:

- Al proceso de producción, está concentrada en los productos y la aplicación del currículo y de la enseñanza; hace énfasis en los resultados del aprendizaje.

- A la lógica de los medios, cuyos significados se concentran en los productos; la enseñanza se justifica de acuerdo con los resultados deseados del aprendizaje.

- En bases técnicas, en las cuales personal experto determina lo que se hace y desarrollan el currículo.

- En la objetividad, en la que las decisiones deben hacerse sobre una base científica sin la influencia de valores personales y prejuicios; por lo tanto, la eficacia y eficiencia están reflejados en los métodos y en los objetivos.

En los documentos curriculares, aquellos que construye la institución, producto del trabajo de un equipo, ofrecen una información ideal en la que podemos inferir: Algunas claves sobre los problemas a los que el currículo respondió y la clase de personal experto, incluye el proceso de desarrollo; una idea clara de lo que el estudiantado aprenderá, objetivos de aprendizaje que se supone que el profesorado enseñará, los contenidos y el orden o secuencia en que deben enseñarse; una idea clara acerca de por qué esos objetivos de aprendizaje y contenidos son importantes, racionalidad o filosofía; alguna guía, en forma de sugerencias o propuestas, de cómo enseñar los objetivos y contenidos, estrategias de enseñanza; indicación de cómo el currículo y el estudiantado deben ser o han sido evaluados y qué resultados obtuvieron; una indicación de si el currículo ha sido implementado, si no lo ha sido, en qué situaciones sería apropiado, si ha sido implementado, qué paso, cuándo fue, etc. 
doi: http://dx.doi.org/10.15359/ree.21-3.3

URL: http://www.una.ac.cr/educare

CORREO: educare@una.cr

El marco conceptual sobre el CDC y lo referenciado en la introducción fue fundamental para definir categorías previas en la investigación (Ver Tabla 1), para la construcción de los instrumentos y desde allí, hacer el análisis de las características del CDC del docente licenciado en química, participante de la investigación.

\section{Metodología}

El estudio investigativo se desarrolló desde el enfoque constructivista cualitativo (Denzin y Lincoln, 1994) porque así se podía orientar y describir el fenómeno social relacionado con el proceso educativo de interés, es decir, la caracterización del CDC del participante, y por el interés de analizar en este proceso, sus signifiados y acciones docentes (frente a su enseñanza, como parte de la caracterización de su (DC), es decir, desde sus perspectivas como agente social; asimismo, esto significó analizar la situación del contexto laboral del profesor, sin pretender generalizar, pero sí profundizar dicha caracterización para la mejora de su enseñanza. La dinámica de la investigación se centró en la participación directa del docente, más que en el presupuesto de tener un sujeto o persona investigada.

De esta manera, la investigación fue holística, porque se estudió una realidad (las aulas de clase en donde el profesor ejerce su enseñanza) desde un enfoque global sin fragmentarla ni seleccionar variables; deductiva porque desde los referentes conceptuales se establecieron categorías previas para el análisis; ideográfica porque se interpretó lo singular del fenómeno analizado (caracterización del CDC del docente al enseñar conceptos de química orgánica). Este proceso consideró criterios de calidad científica cualitativa, en los que se resalta la credibilidad ya que, entre otras, se explicó el origen de las categorías, sus criterios de formulación y la procedencia de los datos; la transferibilidad horizontal porque los resultados se dan en el contexto de investigación sin hacer generalizaciones del caso analizado, recogiendo abundante información y haciendo descripciones densas; y el criterio de confirmabilidad hecho desde los mecanismos de triangulación (de información y datos obtenidos, de técnicas y de quienes investigan).

Como técnica de análisis se usó el análisis documental de la información consolidada de documentos tales como transcripciones de entrevistas semiestructuradas, sistematización de encuestas (instrumento Likert), transcripciones de las observaciones de clase, de las ReCo y los ReP-Ed, de las grabaciones de audio y video y los diseños curriculares del profesor. Se contó con un participante, constituyéndose en el caso a estudiar por ser docente de química, graduado como licenciado en Química y Biología, quien trabaja en una institución de educación superior en Bogotá. El licenciado impartía la asignatura de química orgánica, en un programa que forma ingenieros y técnicos ambientales.

Para este caso, se analizaron las representaciones de contenido (ReCo) y los repertorios profesionales de experiencias didácticas (ReP-Ed) (Mulhall, Berry y Loughran, 2003). Para el análisis, fueron discutidos con el profesor los instrumentos enunciados para su comprensión. Las ReCo 
permitieron revisar las grandes ideas a enseñar, los elementos sobre las decisiones curriculares y la reflexión sobre su práctica docente; los ReP-Ed le permitieron reconocer el tipo de evaluación narrativa de la práctica docente, sus creencias acerca de las respuestas de sus estudiantes y lo sucedido en el desarrollo de las clases, el contenido que le da forma a la enseñanza-aprendizaje y las relaciones que se construyen con el estudiantado (Candela y Viafara, 2014); finalmente, se hizo un análisis documental (AD) de los diseños propios del currículo del docente y de la institución en general.

Además, se usaron instrumentos como el de identificación de las ReCo adaptado por Mora y Parga (2008), un instrumento sobre diseño curricular y un cuestionario Likert adaptado de Mellado (1994); este último permitió determinar el grado de acuerdo o desacuerdo respecto a las acciones docentes y fue aplicado a este personal (constaba de 33 afirmaciones) como al estudiantado (tenía 39 afirmaciones).

Con respecto a los ReP-Ed, que son narrativas de una pieza particular del contenido a enseñar por el profesorado, fue complementado, en primer lugar, con una entrevista semiestructurada sobre la enseñanza que fue adaptada de Mellado (1994); en segundo lugar, se hicieron observaciones de clase, con un mínimo de seis sesiones de acuerdo con los criterios de Cañal (2000) y se hicieron

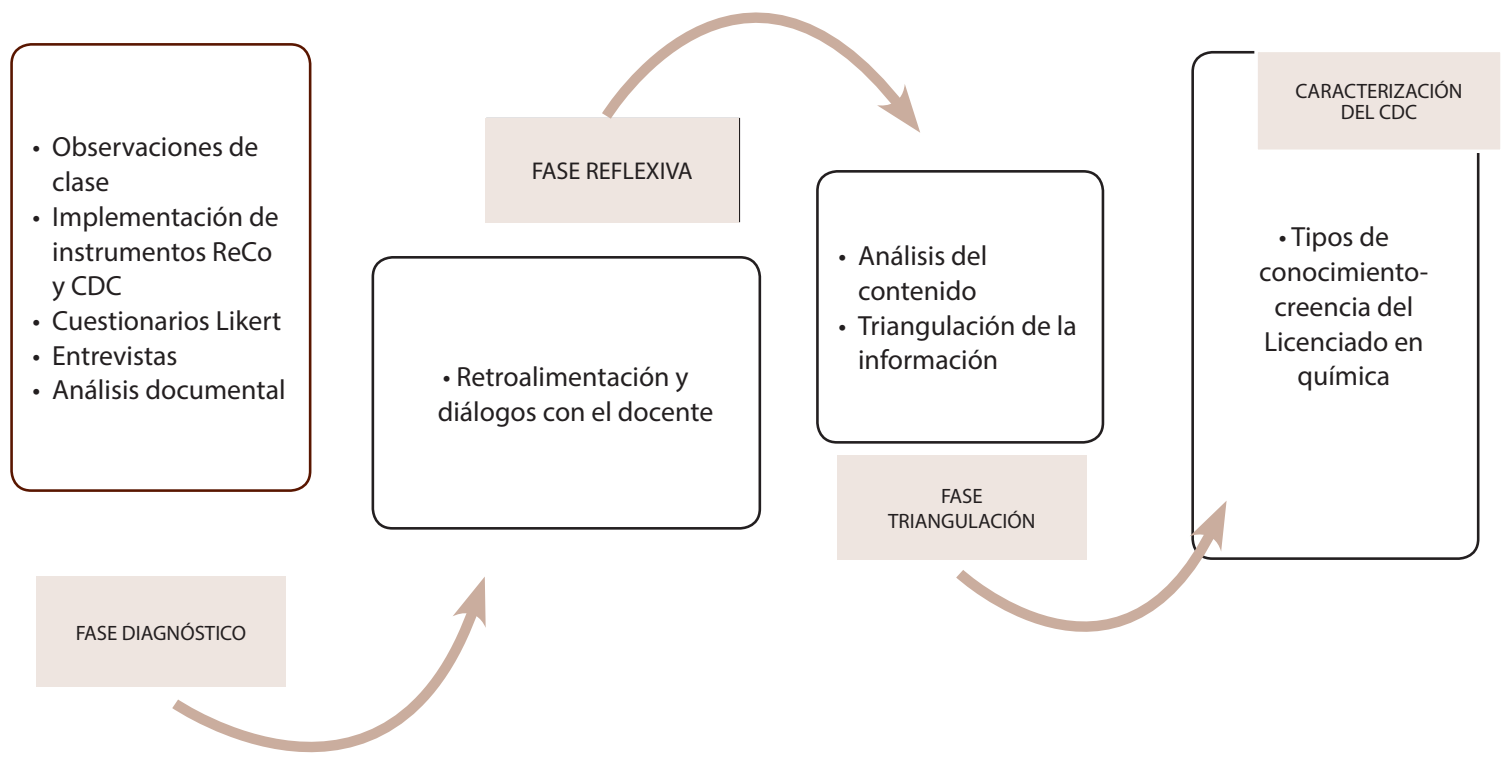

Figura 2: Descripción del proceso metodológico global.

Nota: Elaboración propia.

narraciones orales esporádicas; estas representaciones ofrecen la posibilidad de acceder a la naturaleza holística y compleja del CDC, más allá de las ReCo (Mulhall et al., 2003); estas ReCo proporcionan una pequeña parte del CDC del personal docente, de su razonamiento cuando tiene 
doi: http://dx.doi.org/10.15359/ree.21-3.3

URL: http://www.una.ac.cr/educare

CORREO: educare@una.cr

Tabla 1: Categorías definidas en la investigación para caracterizar el CDC de un profesor de química

\begin{tabular}{|c|c|}
\hline Categoría & Componentes \\
\hline 1. Currículo formal & $\begin{array}{l}\text { Propósitos curriculares, contenidos curriculares, organización } \\
\text { curricular }\end{array}$ \\
\hline 2. Conocimiento disciplinar del contenido: CD & Sustantivo y sintáctico \\
\hline 3. Conocimiento histórico-epistemológico: $\mathrm{CHE}$ & $\begin{array}{l}\text { Mecanismos de producción de conocimiento, obstáculos } \\
\text { epistemológicos, debates y controversias }\end{array}$ \\
\hline 4. Conocimiento psicopedagógico: CPP & $\begin{array}{l}\text { Teorías educativas, conocimiento del currículo, modelos } \\
\text { de desarrollo del aprendizaje del estudiante, concepciones } \\
\text { alternativas del estudiante, metodología de las formas de } \\
\text { organización de grupos }\end{array}$ \\
\hline 5. Conocimiento del contexto escolar: CCE & $\begin{array}{l}\text { ¿Dónde enseña? ¿A quiénes enseña? Normas y } \\
\text { funcionamiento de la institución, procedencia del } \\
\text { estudiantado, configuración cultural, política e ideológica del } \\
\text { entorno de la institución. }\end{array}$ \\
\hline
\end{tabular}

Nota: Adaptada de la Figura 1 de Mora y Parga (2008, p. 64).

acciones exitosas (Acevedo, 2009). Otras fuentes de información utilizadas para la caracterización del CDC docente fueron los documentos diseñados por el mismo profesor, tales como guías de trabajo y planeaciones curriculares; además de los documentos oficiales de la institución, específicamente del currículo guía que facilitó el docente. La descripción de este proceso se resume en la Figura 2.

Las categorías del estudio propuestas para el análisis y que surgieron del referencial teórico se presentan en la Tabla 1, consideran lo que Posner (2004) propone para el análisis del currículo formal y lo que sugieren Mora y Parga (2008) para el análisis del CDC.

La secuencia ejecutada de la información ofrecida por los instrumentos fue: (i) modificaciones discutidas, validadas y aclaradas, (ii) en la implementación se habló con el participante sobre la intencionalidad de estos, (iii) transcripciones de los instrumentos, (iv) interpretación de la información por medio de unidades significativas, (v) formulación de enunciados en correspondencia con las categorías para evidenciar la postura del docente, (vi) formulación de inferencias e interpretaciones de la investigación.

\section{Resultados y su discusión}

Los resultados obtenidos se presentan en la Tabla 2, Tabla 3, Tabla 4, Tabla 5 y Tabla 6, donde se evidencian las principales afirmaciones obtenidas, luego del análisis de los diferentes instrumentos utilizados y del proceso de triangulación de resultados e instrumentos. Es decir, son fruto del análisis documental de las planeaciones curriculares y del análisis de las dinámicas de aula. 


\section{El caso del profesor licenciado en química}

\section{Análisis del conocimiento disciplinar del contenido}

El profesor comparte una visión enciclopedista, compartimentalizadayjerárquica, tendiente hacia esta última, la cual es influenciada por la estructura de las disciplinas que tiene el currículo

Tabla 2: Matriz de afirmaciones de acuerdo con las unidades de análisis para el CD

\begin{tabular}{lcl}
\hline \multicolumn{1}{c}{ Categoria } & Componente & \multicolumn{1}{c}{ Afirmación } \\
\hline Suntantivo & $\begin{array}{l}\text { El profesor sigue esencialmente una interrelación de conceptos } \\
\text { desde la visión de la teoría estructural de la química. }\end{array}$ \\
$\begin{array}{l}\text { del contenido }(\mathrm{CD}) \\
\text { Sintáctico }\end{array}$ & $\begin{array}{l}\text { Sus laboratorios son cualitativos y usa la reactividad química como } \\
\text { un canón de identificación de sustancias químicas. }\end{array}$ \\
\hline
\end{tabular}

Nota: Elaboración propia.

construido por los llamados "expertos". Para el profesor, el estudiantado necesita de experimentos ilustrativos que le permitan avanzar en un contenido específico. El manejo experimental que hace el docente, si bien es satisfactorio, no es el más adecuado ya que recae en las demostraciones; su modelo de enseñanza para estas actividades se basa en organizar pequeños grupos de trabajo, con un modelo tradicional. En sus clases se apoya en la teoría estructural para explicar la química orgánica, lo cual se evidenció al usar la representación de enlaces sencillos, dobles y triples para el carbono, así como la disposición espacial en dos dimensiones. Ver Tabla 2.

Tabla 3: Matriz de afirmaciones de acuerdo con las unidades de análisis para el CHE

\begin{tabular}{lll}
\hline \multicolumn{1}{c}{ Categoria } & \multicolumn{1}{c}{ Componente } & \multicolumn{1}{c}{ Afirmación } \\
\hline & $\begin{array}{l}\text { Mecanismos de producción } \\
\text { de conocimiento }\end{array}$ & $\begin{array}{l}\text { El profesor comparte una visión positivista de la } \\
\text { ciencia, no es reflexivo ante estos elementos ni con } \\
\text { respecto a los contenidos que va a enseñar. }\end{array}$ \\
$\begin{array}{l}\text { Conocimiento } \\
\text { histórico-epistemológico } \\
\text { (CHE) }\end{array}$ & $\begin{array}{l}\text { Obstáculos } \\
\text { epistemológicos }\end{array}$ & $\begin{array}{l}\text { Considera que uno de los grandes obstáculos } \\
\text { epistemológicos es superar errores en las costumbres } \\
\text { y formas de pensar, pero en su práctica evidencia una } \\
\text { necesidad en la enseñanza de superar errores verbales. }\end{array}$ \\
& Debates y controversias & $\begin{array}{l}\text { Considera importante la historia y sus implicaciones en } \\
\text { la enseñanza, aún así su reflexión curricular es pobre al } \\
\text { respecto. }\end{array}$ \\
\hline
\end{tabular}

Nota: Elaboración propia. 
doi: http://dx.doi.org/10.15359/ree.21-3.3

URL: http://www.una.ac.cr/educare

CORREO: educare@una.cr

\section{Análisis del conocimiento histórico-epistemológico}

Como se plantea en la Tabla 3, el profesor expone verbalmente en sus clases los contenidos, realiza talleres y experimentación. Se basa en la observación y el planteamiento de conceptos que apoyen a esta, como imagen exacta de la realidad; dedica su mayor tiempo a la forma en la que el estudiantado asimila y acomoda la nueva información que está recibiendo. Los debates históricos son anecdóticos, por lo tanto, no hay un componente histórico-epistemológico en el desarrollo de la temática.

\section{Análisis del conocimiento psicopedagógico}

El profesor desarrolla el modelo de transmisión-recepción y en otras ocasiones el de descubrimiento, principalmente el primero es el más usado; la práctica experimental es importante para él en la formación de profesionales de ingenierías y de técnicas ambientales, pero se centra en el seguimiento de recetas instruccionales de naturaleza demostrativa de los temas que se exponen en el aula.

Presenta de forma clara y ordenada los contenidos teniendo en cuenta la guía curricular; la evaluación se centra en reproducir o imitar la lista de conceptos tal como lo expuso en clase; la participación del estudiantado se centra en responder y el profesor asume que este aprende por medio de las actividades desarrolladas; además, cree que el estudiantado debe aprender habilidades de investigación. El currículo es un listado de conceptos, combinado con desarrollar destrezas y habilidades. No considera las ideas del estudiantado, al inicio, durante el proceso, ni en su evaluación; su metodología se enfoca en explicar e ilustrar los temas enunciados.

El profesor es claro en las reglas y rutinas que se siguen en el aula de clase y maneja una destreza relativamente alta en este aspecto, en tanto que los procesos cognitivos y científicos los reduce a la exposición de conceptos de manera compartimentalizada y jerárquica, y a la explicación del experimento ilustrativo; las destrezas relacionadas con los objetivos se limitan a lo planteado en el currículo guía y al tiempo que los encuentros con el estudiantado le permitan, este último es muy corto.

Este docente cree que los contenidos son un cuerpo que debe ser transmitido a partir de la explicación verbal, se considera más como científico que como educador, el curso lo modifica según sus valores y creencias, se centra más en ser un profesor que entrega, da y reparte; se percibe que la evaluación ofrece una información que le permite actuar como juez de ello. No resalta aspectos relacionados con la construcción social de las teorías ni de los conceptos. El pensamiento del profesor puede ubicarse en un paradigma de procesoproducto, donde el aprendizaje del estudiantado es el producto y la enseñanza es el proceso. 
Tabla 4: Matriz de afirmaciones de acuerdo con las unidades de análisis para el CPP

\begin{tabular}{lll}
\hline \multicolumn{1}{c}{ Categoria } & \multicolumn{1}{c}{ Componente } & \multicolumn{1}{c}{ Afirmación } \\
\hline & Teorías educativas & $\begin{array}{l}\text { La teoría educativa que utiliza y que ve más viable el } \\
\text { profesor es el de transmisión-recepción. }\end{array}$ \\
& Conocimiento del currículo & $\begin{array}{l}\text { En torno al conocimiento del currículo lo entiende como } \\
\text { un listado de conceptos, aunque también desarrolla } \\
\text { habilidades y destrezas. }\end{array}$ \\
$\begin{array}{l}\text { Conocimiento } \\
\text { psicopedagógico } \\
\text { (CPP) }\end{array}$ & $\begin{array}{l}\text { Modelos de desarrollo } \\
\text { del aprendizaje del }\end{array}$ & $\begin{array}{l}\text { El profesor asume el modelo de aprendizaje del } \\
\text { estudiantado cuando este se transmite elaboradamente, } \\
\text { pero es fuertemente compartido con la idea de que el } \\
\text { estudiantado aprende por medio de una actividad. }\end{array}$ \\
& $\begin{array}{l}\text { Concepciones alternativas } \\
\text { del estudiante }\end{array}$ & $\begin{array}{l}\text { El profesor hace algún diagnóstico superficial, pero no asume } \\
\text { que el estudiantado tiene ideas previas y que puede tener } \\
\text { concepciones alternativas en torno a lo que expone en clase. }\end{array}$ \\
& $\begin{array}{l}\text { Metodología de las formas } \\
\text { de organización de grupos }\end{array}$ & $\begin{array}{l}\text { Utiliza la clase magistral y el experimento ilustrativo, puesto } \\
\text { que asíl le enseñaron a él en su formación como licenciado. }\end{array}$ \\
\hline
\end{tabular}

Nota: Elaboración propia.

Su planificación de la enseñanza está centrada en los contenidos exhibidos en la guía curricular. El profesor evidencia que tiene un pensamiento que guía sus interacciones con el estudiantado en lo práctico del contenido, utilizando la enseñanza tradicional y la disciplina en el aula. Lo anterior se resume en la Tabla 4.

\section{Análisis del conocimiento del contexto escolar}

Sus estudiantes han egresado de colegios públicos; estudian en la universidad porque así pueden tener un buen empleo en lo que se están formando. La mayor parte del estudiantado trabaja en el día y en la noche asiste a las clases; no tiene tiempo para dedicar a sus estudios fuera del aula, por lo que se ve obligado a asimilar los contenidos abordados en el aula. La institución adolece de espacios como laboratorios y materiales didácticos tecnológicos que puedan satisfacer otro tipo de formación o su modificación (Ver Tabla 5). Además, los espacios o encuentros planificados desde el currículo son pocos en número y en tiempo. Al profesor no le parece pertinente modificar las guías curriculares, considera que al ser un profesor de cátedra debe dedicarse a cumplirlas. 
doi: http://dx.doi.org/10.15359/ree.21-3.3

URL: http://www.una.ac.cr/educare

CORREO: educare@una.cr

Tabla 5: Matriz de afirmaciones de acuerdo con las unidades de análisis para el CCE

\begin{tabular}{|c|c|c|}
\hline Categoría & Componente & Afirmación \\
\hline \multirow{5}{*}{$\begin{array}{l}\text { Conocimiento del } \\
\text { contexto escolar } \\
\text { (CCE) }\end{array}$} & ¿Dónde enseña? & $\begin{array}{l}\text { El profesor considera que la institución educativa, } \\
\text { dada su naturaleza y sus normas, impone las } \\
\text { determinaciones psicopedagógicas, los contenidos } \\
\text { y el tiempo para desarrollar los contenidos de la } \\
\text { química orgánica. }\end{array}$ \\
\hline & ¿A quiénes enseña? & $\begin{array}{l}\text { A estudiantes que laboran durante el día, pero } \\
\text { en la noche van a clase; este hecho condiciona la } \\
\text { teoría educativa y los contenidos a enseñar porque } \\
\text { su dedicación es limitada }\end{array}$ \\
\hline & $\begin{array}{l}\text { Normas y funcionamiento de la } \\
\text { institución }\end{array}$ & $\begin{array}{l}\text { No podría hacerse alguna diferencia con la } \\
\text { afirmación de la categoría ¿Dónde enseña? }\end{array}$ \\
\hline & Procedencia de estudiantes & $\begin{array}{l}\text { Los estudiantes y las estudiantes son personas } \\
\text { que trabajan de día y en la noche se dedican a sus } \\
\text { estudios, según el profesor, esta procedencia y su } \\
\text { dinámica determinan las formas de enseñanza y la } \\
\text { evaluación, justificando el modelo de transmisión- } \\
\text { recepción. }\end{array}$ \\
\hline & $\begin{array}{l}\text { Configuración cultural, política } \\
\text { e ideológica del entorno de la } \\
\text { institución }\end{array}$ & $\begin{array}{l}\text { La institución educativa, de acuerdo con su } \\
\text { configuración, enfoca todas sus decisiones } \\
\text { curriculares, para que el profesorado de cátedra } \\
\text { siga formando personal trabajador que cumpla las } \\
\text { expectativas en el sector empresarial. }\end{array}$ \\
\hline
\end{tabular}

Nota: Elaboración propia.

Finalmente, frente al análisis documental de las planeaciones curriculares y del análisis de las dinámicas de aula, tal como se aprecia en la Tabla 6, el profesor tiene un conjunto de conocimientos-creencias tales como: la institución ofrece un repertorio de técnicas de enseñanza, contenidos y tiempos, no aborda otros aspectos en la enseñanza de las ciencias y en especial de la química; el tiempo de dedicación del estudiantado, por las responsabilidades laborales de este, conduce a estudiar pocos contenidos; y la orientación en la formación lleva a tener una persona formada para el trabajo según las necesidades del mercado laboral. 
Tabla 6: Análisis documental y análisis didáctico de la dinámica de aula del profesor

\begin{tabular}{|c|c|c|c|}
\hline Categoría & Componentes & Análisis documental curricular & Análisis didáctico de aula \\
\hline \multirow{3}{*}{$\begin{array}{l}\text { Currículo } \\
\text { formal }\end{array}$} & $\begin{array}{l}\text { Propósitos } \\
\text { curriculares }\end{array}$ & $\begin{array}{l}\text { Los propósitos encierran una formación } \\
\text { para el trabajo, aunque sus objetivos están } \\
\text { orientados a conocer la disciplina, en } \\
\text { especial, al uso y la síntesis de compuestos } \\
\text { químicos. Los objetivos de aprendizaje están } \\
\text { basados en competencias instrumentales, } \\
\text { de trabajo en grupo y comunicativas. }\end{array}$ & $\begin{array}{l}\text { Sus objetivos se basan en transmitir } \\
\text { los conocimientos de reconocimiento } \\
\text { y uso de las sustancias químicas } \\
\text { orgánicas, lo hace con actividades de } \\
\text { laboratorio demostrativo y talleres de } \\
\text { ejercicios de lápiz y papel. }\end{array}$ \\
\hline & $\begin{array}{l}\text { Contenidos } \\
\text { curriculares }\end{array}$ & $\begin{array}{l}\text { Establece una lista de conceptos de } \\
\text { acuerdo con la estructura disciplinar de } \\
\text { la química en donde se pretende enseñar } \\
\text { propiedades físicas, nomenclatura y } \\
\text { aplicaciones (reactividad). }\end{array}$ & $\begin{array}{l}\text { Sigue los contenidos curriculares que } \\
\text { establece la institución y no hace } \\
\text { cambio alguno. }\end{array}$ \\
\hline & $\begin{array}{l}\text { Organización } \\
\text { curricular }\end{array}$ & $\begin{array}{l}\text { Establece un tiempo de dos horas } \\
\text { semanales, propone algunas estrategias de } \\
\text { enseñanza y su organización corresponde } \\
\text { a lo que proponen los libros de química. }\end{array}$ & $\begin{array}{l}\text { Sigue la organización curricular que } \\
\text { propone la institución. }\end{array}$ \\
\hline
\end{tabular}

Nota: Elaboración propia.

\section{Alcance de la caracterización hecha sobre el CDC en la formación de licenciados en química}

El caso analizado deja ver que la formación profesional de este profesor de química (Licenciado en química), ha tenido falencias, las cuales, puestas en el contexto laboral, terminan mostrando a un profesor con un CDC poco deseable y en consolidación, por ello es necesario reflexionar sobre la formación de docentes. Para organizar los elementos en torno a esto, se exponen las siguientes preguntas: ¿Este caso muestra lo desfavorable de la propia formación docente? ¿Qué papel tienen las facultades de educación en la formación de docentes de química? ¿Es pertinente aceptar el paradigma que plantea que para enseñar química solo basta conocer la disciplina -paradigma que desde la didáctica de las ciencias estaba superado-?

- ¿Este caso muestra lo desfavorable de la propia formación docente? Si bien un caso no puede extrapolarse a una población en general como la de docentes con licenciatura, esta investigación revela aspectos de mejora que, en torno a las categorías que componen el CDC, deben fortalecerse en el profesorado licenciado en química, y sobre todo, en los programas mismos de formación; se debe reflexionar profundamente y seguir investigando los conocimientos-creencias del profesorado; se deben considerar 
doi: http://dx.doi.org/10.15359/ree.21-3.3

URL: http://www.una.ac.cr/educare

CORREO: educare@una.cr

elementos dados en las instituciones de educación científica que a veces desvalorizan los conocimientos abordados en la investigación, es decir, lo didáctico de las disciplinas específicas; es fundamental que las líneas de investigación que se desarrollan en este tipo de instituciones contemplen los problemas didácticos que la misma institución encarna, pues la construcción del currículo no está dada por especialistas en la enseñanza de la química, sino por profesionales que se han formado en diversas carreras (ingenierías química, entre otras) por lo que sus conocimientos y creencias son diferentes a las de personal docente profesional con licenciatura en química.

- ¿Qué papel tienen las facultades de educación en la formación de docentes de química? Frente a esto, se asume que las facultades de educación han sido poco constantes en determinar los conocimientos que revela el CDC en este tipo de instituciones de educación superior; deberían ser más pertinentes en realizar análisis que respondan a la necesidad de tener docentes que reconozcan los componentes de este conocimiento, que es el que define al profesorado y lo diferencia de otras profesiones; que se hagan estudios desde la formación misma para ir analizando cómo el profesorado en formación inicial y continua va integrando sus componentes; en todo caso, esas falencias no se resuelvan obligando al profesorado a realizar estudios esporádicos y descontextualizados de sus necesidades (especializaciones y diplomados), que lo que hacen es facilitar el cumplimiento de los requisitos que exige el Ministerio de Educación Nacional (MEN) en torno a una formación y a una enseñanza de una asignatura.

- ¿Es pertinente aceptar el paradigma que plantea que para enseñar la química solo basta conocer la disciplina? El profesor del estudio está enmarcado en una visión institucional, concibe la formación en el CD para formar profesionales -ingenieros y técnicos- aptos para el trabajo; esto encierra una discusión profunda en torno a las intencionalidades curriculares que asume la institución y su impacto con la formación. Hay que comprender que el conocimiento disciplinar de una asignatura tiene características diferentes con respecto a la de otras asignaturas, e incluso de cada carrera profesional. Ser docente profesional hoy, significa poner en acción los conocimientos-creencias desde los componentes de CDC, los que emergen de la práctica; pero que se van construyendo desde la formación en la universidad.

De acuerdo con lo anterior, es necesario trabajar por lograr que el profesorado en formación inicial y continua identifique e integre los componentes del CDC, mostrarse con capacidad reflexiva en las decisiones curriculares, considerando importantes todos sus componentes sin relegar a los otros; es posible que en este proceso, el profesorado se dé cuenta de que tiene preferencia y gusto al enseñar unos contenidos más que otros, porque empieza a identificar sus fallos en la enseñanza, a determinar lo que puede y debe mejorar; evitando asumir, de esta forma, el papel de un profesorado técnico y operario para asumir su rol como profesional de la enseñanza. 
En la formación de las futuras generaciones de profesionales de la enseñanza de la química: (i) la reflexión debe ser un eje fundamental de su práctica docente en torno a los cuatro componentes establecidos en este trabajo de investigación, (ii) la planeación curricular que hace la institución no puede ser la única que se evidencie en la práctica docente, debe ser un construcción colectiva junto con pares docentes, quienes de forma reflexiva planifican desde la postura disciplinar, epistemológica, psicopedagógica y contextual frente a sus procesos de enseñanza, esto llevaría al personal docente a planearla más allá de una secuencia de actividades carentes de argumentación, (iii) la historia, la epistemología, la sociología, etc., de la disciplina deben ser primordiales en la formación profesional del docente, para resolver, desde estas metadisciplinas, preguntas que se deben responder a partir el contexto de la carrera profesional en la que se pretende formar, así, por ejemplo: ¿por qué enseñamos química, cómo se construye esta disciplina, qué impacto ha tenido la química en la sociedad, el ambiente, la política, etc.? (iv) fortalecer la formación psicopedagógica evitando la visión técnica de la enseñanza, integrando, entonces, los conocimientos-creencias del contexto escolar para modificar valores y creencias en torno a la química, reflexión importante para tomar decisiones curriculares en un ambiente tan complejo como el de una institución de educación superior y, sobre todo, por las necesidades de profesionales que la sociedad requiere hoy y que las universidades forman.

Las instituciones de educación superior y las universidades deben aportar en la consolidación de líneas de investigación didáctica; no solo deben exigir una formación postgradual en la docencia, sino convertir a sus docentes en investigadores e investigadoras en las didácticas específicas, de los propios problemas que encarna la institución.

\section{Conclusiones}

Este trabajo permitió caracterizar el CDC de un profesor que enseña química orgánica en una institución de educación superior que forma profesionales de ingeniería ambiental y técnicas ambientales. Dentro de las categorías analizadas se resalta:

- En el conocimiento disciplinar (CD) hay una visión compartimentalizada y jerarquizada de los contenidos y la correlación de conceptos; la organización del contenido no evidencia cuáles son las ideas centrales por enseñar, por lo que no se valorizan ideas centrales de otras. El contenido procedimental se caracteriza por seguir cánones de demostración con base en la reactividad química y de acuerdo con la interpretación de estos, no hay reflexión profunda sobre este. El profesor tiene un conocimiento disciplinar aun poco consolidado.

- En el conocimiento histórico-epistemológico, los mecanismos de producción del conocimiento comparten una visión positivista, enmarcada, en el método científico 
doi: http://dx.doi.org/10.15359/ree.21-3.3

URL: http://www.una.ac.cr/educare

CORREO: educare@una.cr

inductivista; frente a los obstáculos epistemológicos asume la idea de obstáculos como "errores" en las costumbres y formas de pensar de la comunidad científica y que deben ser superados. Los debates y controversias históricas no son utilizados como criterio de planificación curricular, ni como factor dinamizador de la enseñanza.

- Respecto al conocimiento psicopedagógico, la teoría educativa es de transmisiónrecepción; el conocimiento curricular se expresa en un listado de conceptos, en torno al modelo de aprendizaje del estudiantado, está centrado en el activismo. Usa la lección magistral con la modalidad interactiva y el experimento demostrativo o ilustrativo; no aborda la organización de grupos de estudiantes por lo que se hacen trabajos individuales en el aula.

- Con respecto al conocimiento del contexto escolar, el profesor considera que el estudiantado debe combinar su trabajo con el estudio nocturno en la universidad; esto hace que exista una limitación del aprendizaje y el profesor justifica muchas decisiones curriculares con esta razón. De igual manera, percibe que la institución tiene como meta formar personal trabajador competente para el mercado laboral, por ello el currículo se centra en desarrollar habilidades o "capacidades" dentro de un contexto laboral; este tipo de formación determina decisiones curriculares como obviar temas, no profundizar en algunos y hacer actividades demostrativas.

En el análisis documental del currículo, se evidenció la necesidad de formar profesionales en competencias, sin embargo, el docente observado se centra en un listado de conceptos a desarrollaryque propone explicar a través deestrategias de enseñanza comolaconceptualización de estructuras por medio de talleres sistemáticos, exposición de conceptos para asimilación de ideas en relación con el contenido, mecanización de ejercicios como trabajos extras, entre otras; en la práctica, el docente solo se centra en la clase magistral basada en un modelo de transmisión-recepción. Su secuencia de enseñanza está basada en la teoría estructural de la química para explicar hibridación; geometría molecular; enlaces, sencillos, dobles y triples; estructura de grupos funcionales; estructura del carbonilo, aromaticidad y nomenclatura; estos contenidos se corresponden con la propuesta de los libros de texto universitario de química. Es decir, que para este docente no es claro en qué se diferencian los contenidos de la química que forman a cada tipo de profesional, pues todos se asumen por igual.

- Es evidente que para este profesor es fundamental el conocimiento disciplinar de la química para la formación de profesionales en ingenierías y técnicas ambientales, un poco menos lo es frente al conocimiento del contexto escolar, concibiéndolo como la limitante en la enseñanza de contenidos, justifica que la calidad del contenido a enseñar es apropiada para el trabajo que ejercerán, por lo tanto, no aborda otros puntos de vista que puedan ayudarles a ser mejores. 
- El conocimiento-creencias de lo histórico-epistemológico no es considerado en la planificación ni en el desarrollo de las clases, por ello no forma parte de la reflexión con el estudiantado ni como aspecto para mejorar la enseñanza de la química orgánica; esto, de igual manera, fue evidenciado para el conocimiento psicopedagógico, en el que el docente no analiza su modelo de enseñanza, ni las razones por las cuales el contenido que enseña en fundamental, no reflexiona sobre su planeación ni sobre las actividades -que en él predominan-, por ello se acomoda a una enseñanza de transmisión de contenidos reducidos a definiciones.

De acuerdo con lo anterior, el profesor, licenciado en química, considera el conocimiento disciplinar como la base de su enseñanza; es poco o nada reflexivo ante el núcleo temático o las grandes ideas a enseñar; su intencionalidad en torno a este conocimiento-creencias se centra en comprender los contenidos para transformarlos a través del lenguaje escrito y verbal en contendidos enseñables, contenidos definidos curricularmente por la institución. Lo históricoepistemológico de la teoría estructural y su rival la teoría dual, no se enseña, por lo que los aportes hechos desde estas metadisciplinas no son considerados como contenido ni como criterio contextual ni psicopedagógico; por lo tanto, enseña una visión positivista de la ciencia, modificando aspectos como la comprensión de cánones de evidencia experimental. La historia la aísla completamente y le resta importancia en el diseño curricular propio. Esto hace que sea una enseñanza descontextualizada (histórica, epistemológica y socialmente) y, por lo tanto, desarticulada del CD lo cual evita que emerja un mejor CDC.

En cuanto al conocimiento-creencia de lo psicopedagógico, es un saber influenciado por la enseñanza que recibió en su formación como licenciado -modelo de impregnación ambiental-, el de transmisión-recepción, y que ahora expresa en su práctica, aunque es dinámico en su explicación, su intencionalidad se centra en transmitir información. Esto muestra la falta de integración de la formación pedagógica-didáctica con la de su vida profesional y con lo que quiere enseñar, asume que para enseñar basta "saber de química". Frente al conocimientocreencias del contexto escolar, solo considera el tiempo presencial de encuentros extra clase con sus estudiantes, es decir, cuanto más encuentros asume que mejor y más enseña y, por lo tanto, "más aprenden" sus estudiantes; la misma reflexión hace en cuanto a los recursos que la institución ofrece (entre más se tengan, más aprende el estudiantado).

Finalmente, la principal característica del CDC del profesor participante, quien enseñanza conceptos de química orgánica priorizando en el componente disciplinar sobre lo metadisciplinar, contextual y psicopedagógico, limita la hibridación e integración de los componentes que hacen que emerja un CDC más deseable para enseñar conceptos de la teoría estructural. 
doi: http://dx.doi.org/10.15359/ree.21-3.3

URL: http://www.una.ac.cr/educare

CORREO: educare@una.cr

\section{Referencias}

Acevedo, J. A. (2009). Conocimiento didactico del contenido para la enseñanza de la naturaleza de la ciencia (I): El marco teórico. Revista Eureka sobre Enseñanza y Divulgación de las Ciencias, 6(1), 21-46. Recuperado de http://www.redalyc.org/pdf/920/92012998003.pdf

Antibi, A. y Brousseau, G. (2000). La dé-transposition de connaissances scolaires. Recherches en Didactique des Mathématiques, 20(1), 7-40. Recuperado de http://rdm.penseesauvage. com/La-de-transposition-de.html

Candela, B. F. y Viafara, R. (2014). Articulando la CoRe y los PaP-eR al programa educativo por orientación reflexiva: Una propuesta de formación para el profesorado de química. Tecné, Epsiteme y Didaxis: TED, 35, 89-111. Recuperado de https://doi.org/10.17227/01213814.3 $\underline{5 t e d 89.111}$

Cañal, P. (2000). El análisis didáctico de la dinámica del aula: Tareas, actividades y estrategias de enseñanza. En F. J., Perales y Cañal, P. (Coords.), Didáctica de las ciencias experimentales (pp. 209-238). Madrid: Marfil.

Chevallard, I. (1991). La transposición didáctica. Del saber sabio al saber enseñado. Buenos Aires Argentina: Aique.

Denzin, N. K. y Lincoln, Y. S. (1994). Introducting: Entering the field of qualitative research. En: N. K. Denzin y Y. S. Lincoln (Eds), Handbook of qualitative research (pp.1-17). Thousand Oaks, California: Sage.

Garritz, A., Daza-Rosales, S. F. y Lorenzo, M. G. (2014). ¿Transposición didáctica o conocimiento didáctico del contenido o conocimiento pedagógico del contenido? 'A rose by any other name'. Un recuerdo de Sandy Abell. En A. Garritz, M. G. Lorenzo y S. F. Daza-Rosales (Eds.), Conocimiento didáctico del contenido: Una perspectiva iberoamericana (pp. 4-22). Alemania: Editorial Académica Española.

Mellado, V. (1994). Análisis del conocimiento didáctico del contenido, en profesores de ciencias de primaria y secundaria en formación inicial (Tesis doctoral). Universidad de Sevilla, Sevilla. Recuperada de http://fondosdigitales.us.es/tesis/tesis/620/analisis-del-conocimientodidactico-del-contenido-en-profesores-de-ciencias-de-primaria-y-secundaria-enformacion-inicial/

Mora, W. M. y Parga, D. L. (2005). De las investigaciones en preconcepciones sobre mol y cantidad de sustancia, hacia el diseño curricular en química. Revista Educación y Pedagogía, 17(43), 165-175. Recuperado de https://dialnet.unirioja.es/ejemplar/152352 
Mora, W. M. y Parga, D. L. (2007). Tramas histórico-epistemológicas en la evolución de la teoría estructural en química orgánica. Tecné, Episteme y Didaxis: TED, 21, 100-118. Recuperado de http://revistas.pedagogica.edu.co/index.php/TED/article/view/370/375

Mora, W. M. y Parga, D. L. (2008). El conocimiento didáctico de la química: Integración de las tramas de contenido histórico-epistemológicas con las tramas de contexto-aprendizaje. Tecné, Episteme y Didaxis: TED, 24, 56-81. Recuperado de http://revistas.pedagogica.edu. co/index.php/TED/article/view/1083/1092

Mora, W. M. y Parga, D. L. (2014). A portes al CDC desde el pensamiento complejo. En A. Garritz, M. G. Lorenzo y S. F. Daza-Rosales (Eds.), Conocimiento didáctico del contenido: Una perspectiva iberoamericana (pp. 100-143). Alemania: Editorial Académica Española.

Mulhall, P., Berry, A. y Loughran, J. (2003). Frameworks for representing science teachers'pedagogical content knowledge. Asia-Pacific Forum Science Learning and Teching, 4(2), Article 2. Recuperado de https://www.ied.edu.hk/apfslt/v4 issue2/mulhall/index. $\underline{\text { htm }}$

Parga, D. L., Mora, W. M. y Martínez, L. F. (2007). El conocimiento didáctico del contenido como programa de investigación: Un contexto para la enseñanza de la química. Tecné, Episteme y Didaxis: TED, N. ${ }^{\circ}$ Extraordinario: Tercer Congreso Internacional sobre Formación de Profesores de Ciencias [Comunicación Oral N. ${ }^{\circ}$ 97].

Parga, D. L. y Mora, W. M. (2014). EI PCK, un espacio de diversidad teórica: Conceptos y experiencias unificadoras en relación con la didáctica de los contenidos en química. Educación Química, 25(3), 332-342. doi: https://doi.org/10.1016/S0187-893X(14)70549-X

Posner, G. J. (2004). Análisis de currículo (3ª ed.). Bogotá: Mc Graw-Hill.

Shulman, L. S. (1986). Those who understand: Knowledge growth in teaching. Educational Researcher, 15(2), 4-14. doi: https://doi.org/10.3102/0013189X015002004 